\title{
Editorial
}

\section{Polymers from Biomass: Characterization, Modification, Degradation, and Applications}

\author{
Mukund Adsul, ${ }^{1}$ Deepak K. Tuli, ${ }^{1}$ Pratheep K. Annamalai, \\ Dilip Depan, ${ }^{3}$ and Shiv Shankar ${ }^{4}$ \\ ${ }^{1}$ DBT-IOC Centre for Advanced Bioenergy Research, Indian Oil R\&D Center, Sector 13, Faridabad 121007, India \\ ${ }^{2}$ Australian Institute for Bioengineering and Nanotechnology, The University of Queensland, Brisbane, QLD 4072, Australia \\ ${ }^{3}$ Chemical Engineering Department, University of Louisiana at Lafayette, Lafayette, LA 70504, USA \\ ${ }^{4}$ Department of Food Engineering, Mokpo National University, Jeonnam 534-729, Republic of Korea
}

Correspondence should be addressed to Mukund Adsul; madsul@gmail.com

Received 23 February 2016; Accepted 23 February 2016

Copyright (C) 2016 Mukund Adsul et al. This is an open access article distributed under the Creative Commons Attribution License, which permits unrestricted use, distribution, and reproduction in any medium, provided the original work is properly cited.

Polymers from biomass are of prime concern and are the cornerstone in terms of various applications such as biofuels, biomedical, and biocomposite applications. Recently, concerns on the environmental pollution and exhaust of natural resources caused by the nonbiodegradable petroleum-based plastics materials have attracted attention on the development of environmentally benign polymers for their applications in various industries and other value added utilities. Renewable and abundantly available biopolymers are the most viable alternative for the production of green materials in the near future. In order to secure the sustainable development for exponentially growing population, the increasing demands for the light-weighted high performance materials and growing concerns over environmental impact of the materials have compelled academic and industrial researchers to develop new materials from alternative or renewable resources. Renewability of resources depends on the availability and life cycle of the raw materials. In recent decades, polymeric materials from renewable biological resources such as plants, marine animals, and microbial organisms have increasingly gained the attention of researchers. The polymers which are derived/extracted from the most widely available biological renewable resources (agricultural plants, marine animals, and microorganisms) are called "biopolymers." These polymers are produced as biomass or byproduct during the growth cycles of organisms. Biopolymers or renewable polymers such as cellulose, lignin, starch, pectin, chitin, and xylan are the abundantly available polymers in nature in the form of plant biomass or other biological sources. Their importance for various applications (biofuels, nanobiocomposites, biomedical, etc.) has been analyzed for many years and still continued. There are different ways to convert these biopolymers into various chemicals, fuels, and materials for the benefit of our society. Still there are challenges to develop new methodologies or improved processes for efficient and economic utilization as well as conversion of these biopolymers.

At present few polysaccharides such as cellulose, starch, and xylan have tremendous applications in various fields such as nanoscience, biorefineries, and composites materials. Some biopolymers are yet to be exploited more such as lignin. Their separation, degradation, and aromatic nature make them more complicated but still have importance. Almost all biopolymers are degraded by microorganism by producing enzymes. They can be also degraded by chemical catalysts (e.g., solid acids) to make sugars. Recently researchers succeeded in preparing the nanoparticles of biopolymers such as cellulose and chitin, which increases their scope in nanosciences. Biological or chemical degradation of biopolymers into their simple forms (e.g., sugars) makes them applicable in biological fermentation for production of value added chemicals or fuels. These biopolymers also have medical applications such as drug delivery and tissue engineering.

Biopolymers have great potential in the growing commercial plastics market with the global production capacity 
of bioplastics at about 1,161 metric tons in 2011, and this has been expected to increase up to 5,779 metric tons by 2016, as indicated by recent market survey. They are regarded as environmentally friendly materials not only due to their source but also due to their inherent biodegradability in many cases. There is also a significant portion of polymers produced from biological resources that are not biologically "degradable" according to the internationally accepted standards. In order to translate these biopolymers into real applications, there appear to be significant obstacles including poor processability, poor properties (mechanical properties, thermal stability, water absorption, and barrier properties), and performance in comparison to their synthetic counter parts. These obstacles have motivated many research activities in the field to process and evaluate materials fully or partially based on the renewable resources.

This special issue portrays the recent and significant research activities occurring around the globe, on the utilization of potential biomass into polymeric materials. Some microbial biomass based on microbiologically produced polymers (alginate) is also highlighted in this special issue along with plant based polymers. Application of chitosan in probiotic culture formulations, use of oil palm biomass for preparations of bioplastic fertilizer composites and use of starch or other carbohydrates for preparation of composite materials, and so forth are few highlights for this special issue. Being readily available, these polymers may need significant improvements for end applications. For this, novel technologies and strategies are currently being researched. Similarly, various investigations to study the thermal and environmental degradation were carried out and are highlighted in this special issue. Interestingly, renewable polymers can be also used as a binding material for construction purpose. Underdeveloped countries may harness benefits as these countries have enormous natural resources, and this aspect has been highlighted in the special issue. Apart from processing products, the biopolymers were also used to release biologically relevant materials in a controlled manner.

\section{Acknowledgments}

We acknowledge all the authors for their valuable contributions and the reviewers for their support and constructive critiques in making this special issue possible.

Mukund Adsul

Deepak K. Tuli

Pratheep K. Annamalai

Dilip Depan

Shiv Shankar 

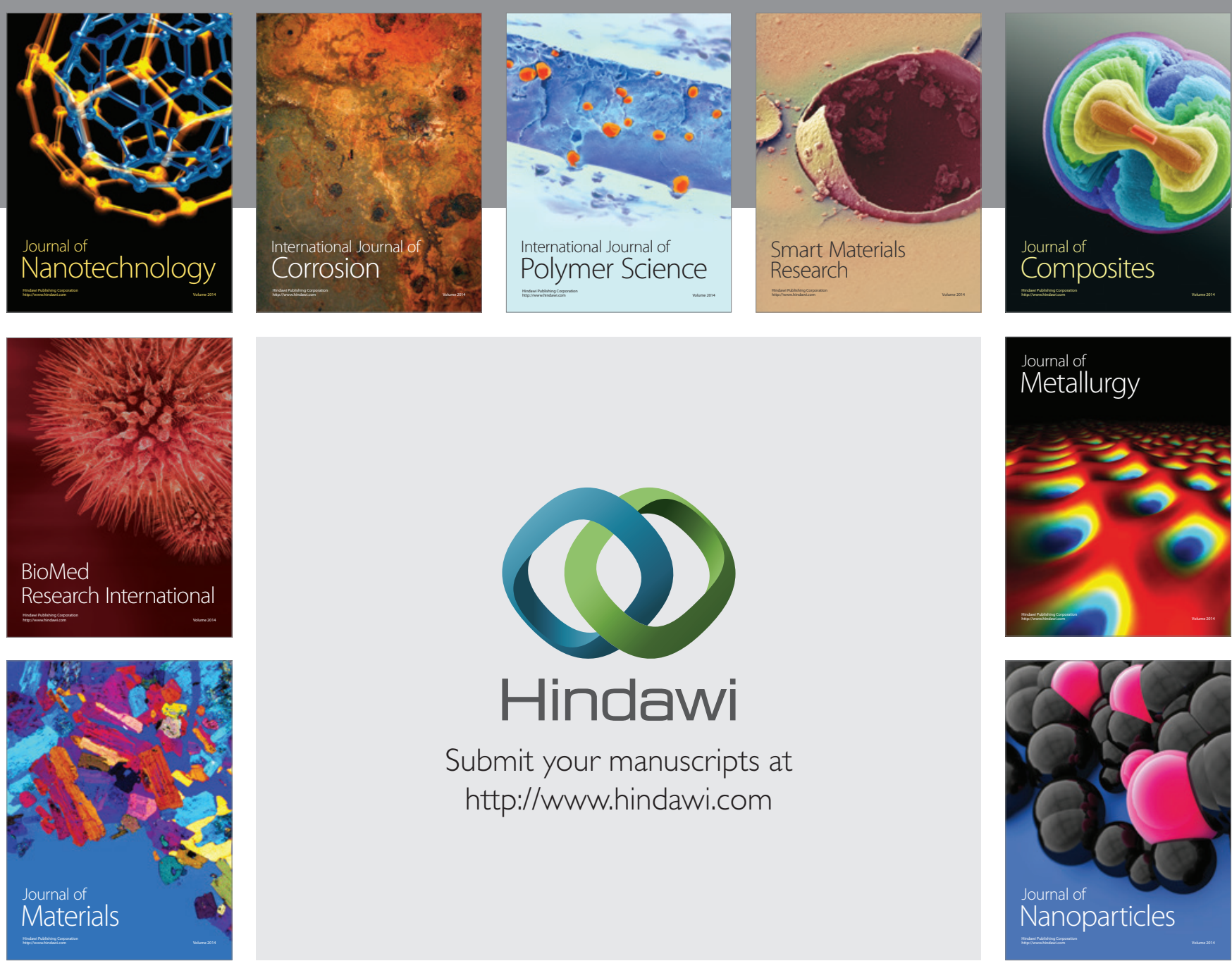

\section{Hindawi}

Submit your manuscripts at

http://www.hindawi.com

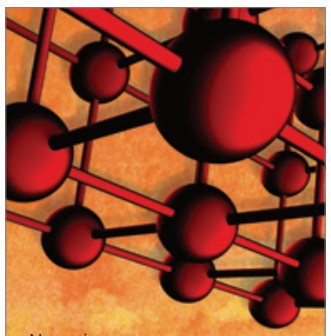

Materials Science and Engineering
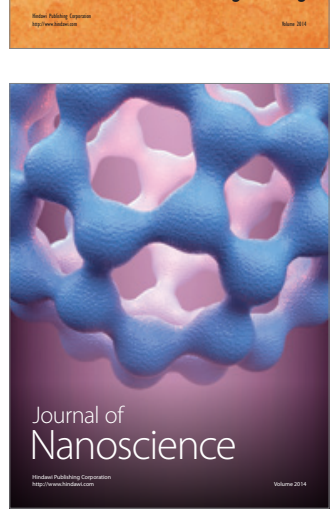
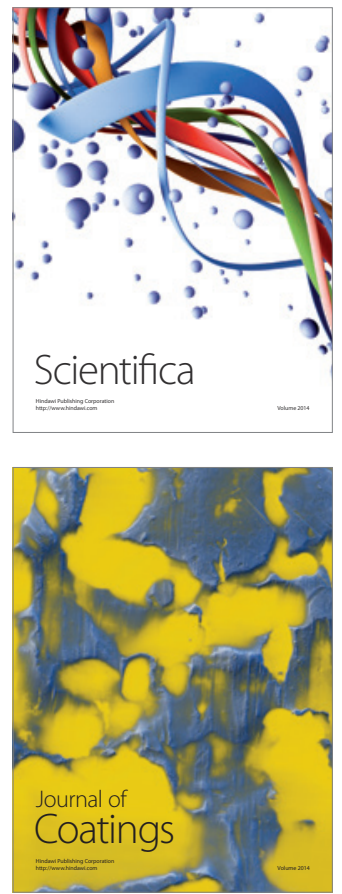
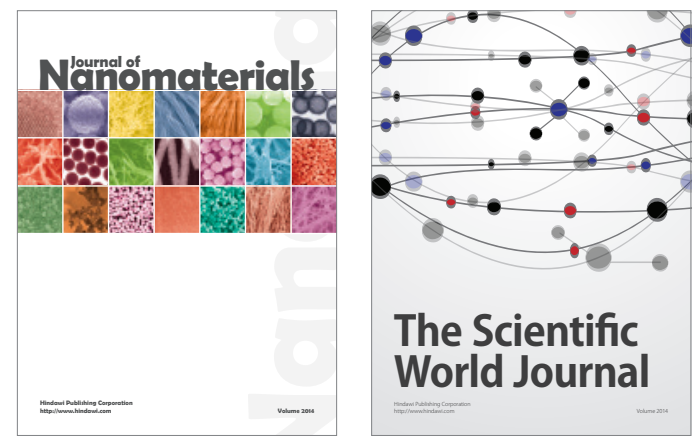

The Scientific World Journal
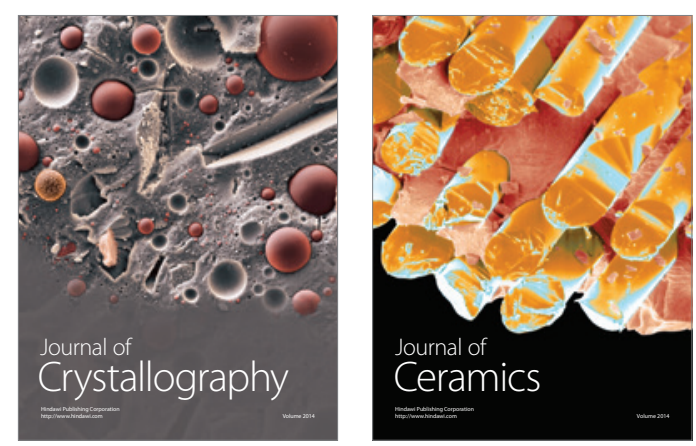
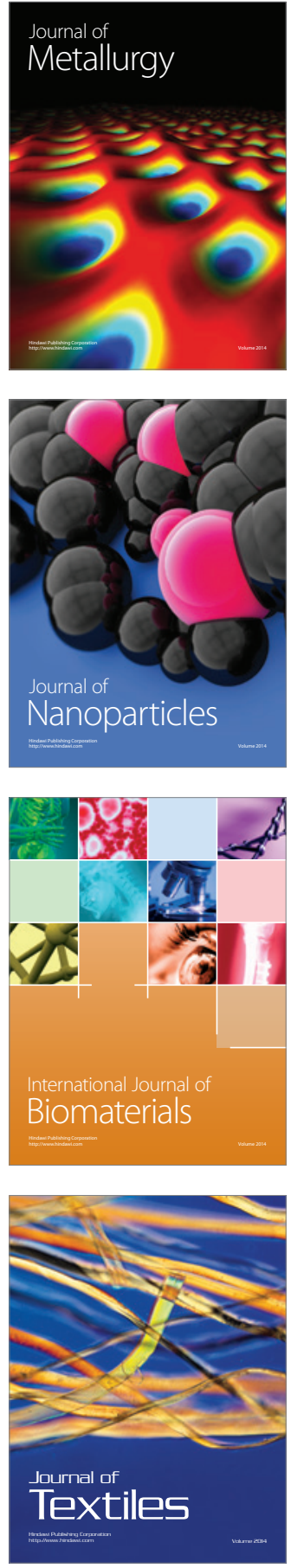